\title{
Impacto de la retención estudiantil en el desarrollo institucional
}

\section{Impact of student retention on institutional development}

\section{Daniel Claudio Perazzo Logioia ${ }^{*}$, Douglas Armando Quintero Bastardo², Ledys Lisbeth Jiménez González ${ }^{3}$}

1.Vicerrector del Instituto Superior Tecnológico Particular Sudamericano, Cuenca. Email: vicerectoradoits@sudamericano.edu.ec ORCID ID: https://orcid.org/0000-0003-3089-2225

2. Coordinador de Seguimiento a Egresados y Graduados del Instituto Superior Tecnológico Particular Sudamericano, Cuenca. Email. Email: daquintero@sudamericano.edu.ec ORCID ID: https://orcid.org/0000-0002-1437-2732

3. Coordinadora de Investigación del Instituto Superior Tecnológico Particular Sudamericano, Cuenca, Ecuador. Email: investigacionits@sudamericano.edu.ec ORCID ID: https://orcid.org/0000-0001-8743-1206

Para Citar: Perazzo Logioia, D. C., Quintero Bastardo, D. A., \& Jiménez González, L. L. (2020). Impacto de la retención estudiantil en el desarrollo institucional. Revista Publicando, 7(25), 193-201. Recuperado a partir de https://revistapublicando.org/revista/index.php/crv/article/view/2118.

Resumen: El índice de Desarrollo Humano, en adelante IDH, actualmente es un indicador utilizado con el propósito de medir el avance de un país a partir de tres dimensiones principales, ellas son: la posibilidad de disfrutar de una vida larga, pero a la vez saludable, el nivel de vida que sea considerado como digno de todo ser humano y el nivel de acceso que la población tenga a la educación. Anteriormente, un país en desarrollo se traducía en la cantidad de territorio que poseía, en riqueza mineral y forestal, en manejo de maquinaria, en la cantidad de fábricas a su disposición. Hoy en día el crecimiento económico depende estrictamente de la preparación profesional que tengan sus habitantes, ya sea básica, técnica o universitaria, y, más aún, su formación continua es imprescindible en el ámbito económico y desarrollo de una nación. Es por ello que muchos países se esfuerzan en aumentar su potencial y crecimiento educativo en todos sus vértices; ejemplo de ello son: Finlandia, Noruega, Japón, Estados Unidos, entre otros, que se enfocan en establecer estrategias educativas avanzadas para que sus estudiantes se formen con los más altos niveles de preparación, para luego, incorporarlos al sector productivo contribuyendo a fortalecer la producción empresarial y por ende su economía. El presente artículo tiene como objetivo analizar la relación existente entre el impacto de la retención estudiantil y el desarrollo institucional, lo cual determina la complejidad existente para el crecimiento de las instituciones educativas.

Palabras clave: Desarrollo institucional, calidad, eficiencia terminal, retención estudiantil.
Abstract: The Human Development Index, hereinafter HDI, is currently an indicator used with the purpose of measuring the progress of a country from three main dimensions, such as: the possibility of enjoying a long but healthy life, the standard of living that is considered worthy of every human being, and the level of access that the population has to education. Previously, a developing country was translated in the amount of territory it owned, in mineral and forest wealth, in machinery management, in the number of factories at its disposal. Today's economic growth strictly depends on the professional training of its inhabitants, being it basic, technical or university, and, furthermore, their continuous training is essential in the economic field and development of a nation. That is why many countries strive to increase their educational potential and growth in all its corners. Examples of this are Finland, Norway, Japan, the United States, among others, which focus on establishing advanced educational strategies so that their students are trained with the highest levels of preparation, and then incorporate them into the job emporium, helping to strengthen business production, and therefore to their economy. This article aims to analyze the relationship between Institutional Educational Growth and the student retention capacity generated by each Institution, which determines the existing complexity for the growth of educational institutions.

Keywords: Institutional growth, quality, terminal efficiency, retention, desertion. 


\section{INTRODUCCIÓN}

$\mathrm{E}$ 1 presente artículo tiene como objetivo analizar la relación existente entre el impacto de la retención estudiantil con el desarrollo institucional, considerando a su vez, la capacidad de retención estudiantil que generan las instituciones de educación superior. Existen diversas dimensiones que deben ser abordadas desde la perspectiva de la formación educativa en todos los niveles del sistema educativo, lo que implica la consideración de una serie de aspectos que deben analizarse, con objeto de medir la calidad y pertinencia social, ya que ello fundamenta las políticas educativas institucionales y el apoyo financiero para la consolidación y el desarrollo de las infraestructuras educativas, los programas de formación docente, la calidad de los servicios educativos, el apoyo a las familias y comunidades en general, corresponsabilidad entre los agentes educativos y el Estado.

Para comprender las implicaciones que tiene la retención estudiantil, con respecto al desarrollo institucional, es necesario definir el término retención que se deriva del latín retentio, onis, que significa acción y efectos de retener. Lo que significa a juicio de Pineda, et al (2011) que un programa de retención estudiantil comprende un conjunto de acciones y estrategias que aborda una institución educativa para garantizar el acompañamiento al estudiante durante su trayectoria académica y por ende lograr una inserción sociolaboral exitosa.

En consecuencia, lleva un proceso de seguimiento y corresponsabilidad permanente en todos los ciclos formativos. Para referirse al término retención estudiantil y desarrollo institucional la Agencia Interamericana de Cooperación y Desarrollo, conocida por sus siglas: AICD señala que el mismo consiste en un programa de fomento y permanencia para garantizar el éxito académico. Es decir, fortalecer el desarrollo del estudiante. Sin embargo, para garantizar el desarrollo educativo institucional y la capacidad de retención estudiantil se analizan aspectos referentes a las políticas educativas del Estado ecuatoriano, los planes y programas de atención a la retención estudiantil y el impacto que puede generar en una organización con fines a la atención de la demanda estudiantil en todos los ciclos educativos; con especial consideración en la educación de tercer nivel.
Swail, et al. (2003) agrupan los programas destinados a apoyar al estudiante en su tránsito por la educación superior en cinco grandes categorías, que se denominan programas de reclutamiento y admisión, programas de apoyo financiero, programas de servicios académicos, programas relacionados con el currículo y la instrucción, programas de servicios estudiantiles.

El tema de la inclusión, la equidad, la atención a la diversidad y la calidad de la educación, se ha venido tratando en congresos, reuniones y cumbres, como por ejemplo en la Segunda Cumbre de las Américas (1998) donde se afirma que:

(...) la creación de condiciones para que toda la población tenga la oportunidad de recibir servicios educativos con calidad, reduciendo de manera apreciable los efectos que se derivan de la desigualdad social y económica, la discapacidad, la discriminación étnica, cultural y de género, se puede afirmar que un componente central de la calidad es la equidad. En nuestra región, América Latina, equidad y calidad están indisolublemente ligadas una a la otra. Sólo es posible mejorar la calidad con políticas de equidad y sólo mejorará la equidad centrando la atención y los recursos en posibilitar aprendizajes de calidad.

Frente a los planteamientos realizados anteriormente cabe preguntarse:

¿Qué estrategias pueden aplicarse para promover el crecimiento institucional educativo y garantizar la retención estudiantil?

Esta interrogante busca ser despejada a lo largo de la presente investigación documental y con información de primera mano, de tipo descriptiva argumentativa que tiene por objetivo presentar un conjunto de acciones dirigidas a garantizar la retención estudiantil en las instituciones educativas.

\section{DESARROLLO}

Cada nación establece en sus leyes, la gratuidad y obligatoriedad de la educación que se imparte, cada institución educativa trata de introducir una enorme 
tecnología y recursos educativos para lograr el máximo rendimiento, dan facilidades para obtener becas de estudio, premian a sus estudiantes que logran altos rendimientos, incentivan a la creatividad, al esparcimiento y confort de los alumnos de cada escuela, en cada universidad, todo con una finalidad de lograr: "Grandes profesionales" que ganen "Premios Nobeles".

En el Ecuador, las universidades y demás institutos de educación superior están sufriendo un crecimiento muy lento con respecto a otros países, sin embargo, se ha notado en los últimos años algunas de ellas, sobre todo instituciones particulares, la introducción de políticas educativas que han contribuido a mejorar el nivel y confort del estudiante para aumentar el producto final.

Por lo tanto, es importante identificar que el crecimiento institucional, como un elemento del impacto en la retención de los estudiantes, aunado al desarrollo de cada institución, obedece no a un capricho, sino a un crecimiento económico de la sociedad: "Se puede definir como un cambio planificado y sistemático coordinado y asumido por la institución en la búsqueda del incremento en los niveles de calidad, equidad y pertinencia en los servicios ofrecidos" (Viera, 2016 p.23).

Carlos Gómez Figueroa en la XVI Asamblea General Ordinaria de la ANUIES, celebrada en la ciudad de Querétaro en 1975 dice:

Actualmente las instituciones de educación superior se enfrentan a un proceso de cambio que, refiriéndose a sus aspectos cuantitativos, se manifiesta en una expansión de la capacidad institucional; en lo cualitativo está estrechamente relacionado con la transformación de la estructura de las propias instituciones. Para prever y regular el cambio, se ha pensado en la conveniencia de disponer de una metodología que considere el estado actual de la institución, los objetivos por alcanzar, las políticas y programas a implantarse, así como los requerimientos y limitaciones del ámbito en el que está situada. Los objetivos, políticas y programas constituyen el modelo de crecimiento institucional con el cual se configura el tipo de institución educativa que pretende lograrse. Un modelo así deberá constar de lineamientos de carácter general que permitan a cada casa de estudios elaborar una versión particular del modelo atendiendo a su circunstancia específica (Gómez, 2000).

Este crecimiento va en concordancia con verdaderos cambios futuros, prospectiva de crecimiento institucional, detectados a nivel pasado y nivel presente. El crecimiento para una institución educativa sería verificar, si las estrategias utilizadas en su funcionamiento fueron eficientes o no, si la calidad de sus egresados cumple con las expectativas que exige la sociedad productiva, si el nivel de preparación está acorde con los cánones empresariales.

El interés estudiantil de continuar sus estudios y formarse con alto nivel depende mucho en cuanto a estudiantes matriculados y estudiantes retirados, es decir, del índice de eficiencia terminal de las cohortes, para esto hay que diagnosticar las causas de sus retiros, la calidad educativa, la equidad y si se aplicó una verdadera estrategia de retención de esos estudiantes, además a nivel presente si se han hecho también cambios estratégicos en innovación tecnológica, revisión de las carreras y dentro de ellas los programas, para que sean apetecibles al futuro profesional, revisar si existe un alto nivel de confort, de oportunidades de seguir sus carreras y de verificar si hay un seguimiento efectivo como estudiante, como egresado y como graduado en situación profesional.

Todos estos factores influyen en el crecimiento de cualquier empresa educativa particular. Los costos de mantenimiento de un estudiante influyen notablemente en el crecimiento futuro de la institución. Creemos que un diagnóstico de la institución nos daría un enfoque inicial de cómo ha ido creciendo o decreciendo esta institución educativa. Un primer paso sería verificar la eficiencia terminal por carrera, que según Larrea de Granados $(s / f, p .2)$ es que:

Unos de los problemas que enfrenta el sistema de educación superior tiene que ver con la tasa de eficiencia terminal que se expresa en estudiantes que se titulan tardíamente o desertan de su formación profesional. Solo 11 de cada 100 alumnos de universidades públicas se gradúan con su correspondiente cohorte.

Esto reflejará el índice de deserción, lo cual nos daría el índice de crecimiento estudiantil. Las deserciones generan los factores causales de dichos retiros, el por qué un estudiante quiere retirarse y cuáles han sido las estrategias para retenerlo, que directamente nos conllevaría a establecer si dicha estrategia fue efectiva o no, dando como resultado el índice de retención estudiantil que incidiría en un verdadero beneficio económico a la institución. Claro está que este aspecto positivo no influye directamente en el crecimiento, pero sí en equilibrarlo.

Ahora: ¿Qué se podría hacer para aumentar el crecimiento educativo? 
En primera instancia tomar muy en cuenta éste índice de retención "I.R", donde muchas universidades e institutos de educación superior profundizan en las causas de deserción y las vapulean a través de estrategias efectivas, que logran mantener firme la retención del estudiante y su fijación de culminar la carrera.

Muchos motivos pueden producir en el estudiante novato, ese ímpetu de abandonar, razones como: su situación económica (familiar), la salud, las enfermedades terminales, la dificultad para llegar al sitio de estudio, falta de alimentación, desnutrición, situación laboral, situación emocional, situación psicológica, falta de estímulos, aburrimiento, falta de incentivos y actividades complementarias, programas y docentes tediosos, reprobación de los cursos por profesores de línea dura, fobias, incapacidades físicas, discriminación, falta de equidad, estrategias pedagógicas mal utilizadas, falta de afectividad grupal, desanimación intelectual, carencia de actividades de recreación, escaso apoyo médico, baja autoestima, falta de preparación escolar, docentes no pedagogos y sin preparación, falta de apoyo didáctico, no pertenencia a la institución, poca vinculación con las empresas y la sociedad, inexperiencia al entrar al campo laboral, falta de criterios y análisis para enfocar un problema.

Tomando en cuenta la mayoría de estos factores, se puede preparar un plan de mejoramiento que induzca a reparar todas estas falencias y contribuir así, a la solución que permita el crecimiento positivo de la institución educativa. Hoy día las universidades y academias de educación superior están haciendo muchos esfuerzos e invirtiendo grandes cantidades de recursos para lograr que todos estos aspectos se solventen con el único objetivo de lograr mayor matriculación y retención educativa.

Encontramos entonces universidades nacionales dentro del rankings QS a nivel mundial como la Universidad de San Francisco de Quito en el puesto 55, hecho que implica la gran inversión realizada tanto en infraestructura, academia y bienestar estudiantil.

Esto no quiere decir que debemos construir en todos los rincones del Ecuador, universidades tipo Harvard en la ciudad de Cambridge, o La Sorbona en París, para tener una buena educación y formación universitaria, todo lo contrario, lo que si debemos tomar en cuenta son las mínimas estrategias aplicadas por estas instituciones para despertar un gran interés en el futuro estudiante que quiere ser un buen profesional, ya sea la universidad pública o privada, grande o pequeña, que esté en el oriente o en el occidente, todo en una sola búsqueda: Incentivar el crecimiento institucional educativo y aplicar la retención estudiantil.

Para lograr estos objetivos es necesario en primera instancia, la creación de un equipo evaluador que se encargue de realizar un diagnóstico de todos los recursos académicos y de la infraestructura con que cuenta cada institución. En segundo lugar, aplicar instrumentos de recolección de datos para conocer tanto en estudiantes, como en el personal académico, administrativo y de servicio, el grado de satisfacción que tienen con respecto al rol que les compete en el ámbito universitario.

El tercer recurso es construir una base de datos con estadística que sirva como estructura para establecer el cálculo de los índices de eficiencia terminal, causas de deserción, falencias en infraestructura y otras variables necesarias para realizar el seguimiento estudiantil, como el seguimiento a graduados y egresados y su desenvolvimiento laboral en el campo de trabajo. A través de estos estudios ya se contaría con material suficiente para establecer procedimientos y estrategias de retención estudiantil que incidirían definitivamente en el crecimiento educativo.

\section{Estrategias para la Mejora Continua de la Retención Estudiantil: el caso del Instituto Superior Tecnológico Particular Sudamericano de la ciudad de Cuenca}

Para detectar las estrategias a aplicar en cuanto a la retención de los estudiantes en la institución, se consideraron dos aspectos críticos de la calidad educativa, en lo que respecta al compromiso del estudiante: en primer lugar, la cantidad de tiempo y esfuerzo que dedica a sus estudios y cómo la institución despliega sus recursos y organiza el currículo y otras oportunidades de aprendizaje para su motivación. Por lo tanto, consideramos que el compromiso es un proceso incluyente.

¿Por qué es tan importante desarrollar estrategias de retención de los estudiantes en la institución?

Porque el compromiso del estudiante mejora la calidad de la experiencia académica, contribuye a aumentar la retención estudiantil y fortalece la relación estudiante-institución. 
Estos son factores esenciales que debemos considerar en el instituto. "Enlazar a los estudiantes en nuestras instituciones de múltiples maneras positivas les ayuda a permanecer con nosotros, a aprender con más eficacia, a disfrutar de su experiencia de estudiante, y a prepararse para la vida después de la Educación Superior" afirma Deneen (citado en UPlanner, 2019).

Según la U-Planner (2019) en su guía ¿Cómo Mejorar la Retención Estudiantil en la Educación Superior en EE. UU.? Nuevas Estrategias, Enfoques y Tecnologías para atraer y retener a los Estudiantes, existen doce razones de por qué los estudiantes renuncian a sus estudios superiores:

1) Desigualdad en conocimientos y habilidades académicas formales.

2) Falta de conocimiento informal sobre cómo ser un estudiante de tercer nivel.

3) Insuficiente desarrollo de habilidades de autocontrol.

4) Deterioro de la autoeficacia y flexibilidad.
5) Una mentalidad que cree en habilidades más flexibles que fijas.

6) Incapacidad para retrasar la gratificación. Deterioro del juicio ético.

7) Separación del entorno de educación superior.

8) Falta de interés en los cursos.

9) Problemas en la trayectoria académica.

10) Problemas psicológicos.

11) Preocupaciones financieras.

Considerando estos aspectos, es necesario contar en las instituciones con inversión e investigación, ambas son primordiales. Soluciones digitales como softwares de gestión de planificación estratégica de compromiso estudiantil, pueden hacer una contribución decisiva en este sentido.

Es necesario considerar en la actualidad los vínculos institucionales para la retención estudiantil, los cuales se observan en la siguiente figura (1):

Figura 1:

Vínculos Institucionales para la Retención Estudiantil

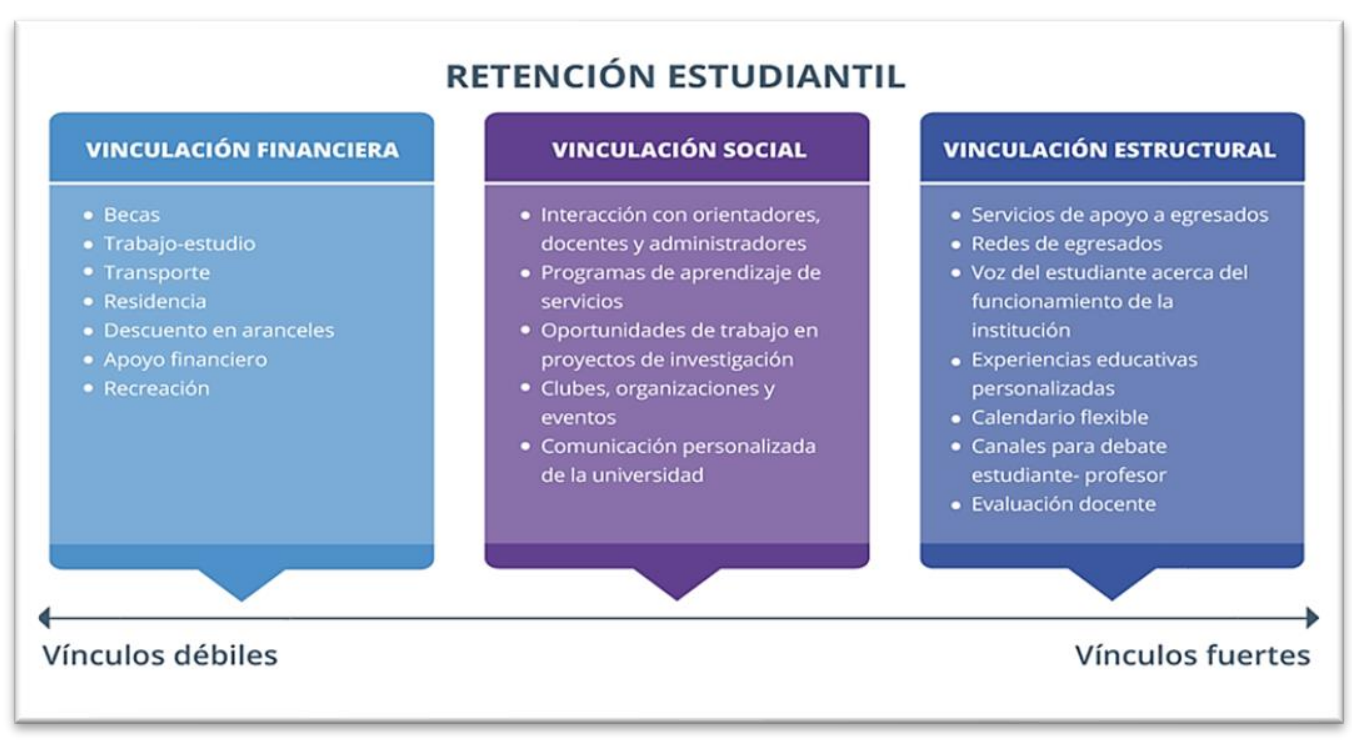

Fuente: Diseñado por U-Planner, citando a Ackerman \& Schibrowsky, 2007. 
La institución a través del área de Psicología dependiente de la Coordinación de Bienestar Institucional refiere la situación en el ciclo marzo-agosto 2019 sobre el número de alumnos que presentan deserción académica en el ciclo por los motivos que pueden observarse en la Tabla 1:

\section{Tabla 1:}

\section{Motivos de deserción académica}

\begin{tabular}{lll}
\hline Motivos & Número de alumnos & Porcentaje \\
\hline Laboral & 5 & $16 \%$ \\
Académico & 4 & $13 \%$ \\
Económica & 5 & $16 \%$ \\
Personal & 10 & $31 \%$ \\
Económico y Personal & 1 & $3 \%$ \\
Académica y Económica & 1 & $3 \%$ \\
No contestan & 6 & $19 \%$ \\
TOTAL & 32 & $100 \%$
\end{tabular}

Fuente: Elaboración del área de Psicología para el ciclo marzo-agosto 2019, con base a una matrícula total de 947 estudiantes.

Considerando los motivos de deserción que figuran en la tabla es que se plantea el Programa de Retención Estudiantil, identificando los procesos de acompañamiento individual y grupal aplicados a través del Programa Institucional de Tutorías del año 2018 para

responder a las necesidades de nuestros estudiantes, permitiendo así, la mejora continua de la oferta educativa, disminuyendo las brechas de segregación e inequidad tanto sociales como educacionales. Pese a esto, aun cuando resulta ser un avance significativo, es insuficiente considerando aquellos estudiantes que provienen de contextos de mayor vulnerabilidad social y del sistema de educación público y subvencionado, quienes presentan mayores factores de riesgo para un potencial abandono (Barba y Heras, 2020 p. 1).

No sólo es importante detectar la problemática, sino desarrollar y aplicar estrategias de retención estudiantil para la mejora continua institucional. Algunas de ellas las retomamos del documento "Estrategias de Deserción para los Estudiantes del Instituto Superior Tecnológico Particular Sudamericano de la ciudad de Cuenca, para las Carreras de Marketing, Diseño Gráfico, Desarrollo de Software, Gastronomía y Turismo, 2019" (Instituto Superior Tecnológico Particular Sudamericano, 2019):

1.- Actividades Complementarias: la realización de eventos donde se involucre al estudiante, incentiva el interés de ellos hacia la institución. Jornadas deportivas, banda show, fiesta de fin de año, exposiciones, casa abierta con premiación, danza, grupos musicales, grupo de teatro, tertulias y conversatorios literarios, sala de lectura, encuentros de graduados, muestra de cine, concursos de diseño con premiación, grupo coral, festivales de canto, concursos de poesía, fotografía, participación en la radio institucional, 
radiodifusión, títeres, viajes y turismo, entre otros.

2.- Bienestar Institucional: Da apoyo moral, jurídico y de salud al estudiante estableciendo una estrategia de retención permanente para la institución. Ello a través del programa de becas, ayudas económicas, servicio jurídico, servicio psicológico, tutorías, seguro de vida, ayuda al emprendimiento, inserción laboral, beca de trabajo, vinculación con empresas, pasantías, seguimiento al graduado, bolsa de trabajo. Hay otros aspectos que son parte del bienestar institucional que gradualmente la institución deberá ir integrando como es la figura del trabajador social y el servicio médico, entre otros.

\section{3.- Educación Continua: Educación formativa permanente} que ayuda al estudiante a seguir formándose después de haberse graduado de la institución. Cursos cortos, cursos largos, talleres de aprendizaje, tutoriales, formación empresarial, cursos de posgrado, dominio de idiomas, maestrías tecnológicas, convenios universitarios nacionales e internacionales, intercambios culturales, charlas motivacionales.

\section{4.- Estrategias de Aprendizaje: Continua evaluación de los} procesos de calidad y de los planes y estrategias de aprendizaje utilizadas en clase. Con la finalidad de realizar cambios efectivos en el proceso de enseñanza-aprendizaje y evitar los esquemas de enseñanza tradicional utilizados por algunos docentes, para dar paso a estrategias innovadoras que incentiven el conocimiento y el aprender haciendo.

\section{5.- Mejoras en la Infraestructura y la Ergonomía. Un sitio} confortable y acorde con las tareas realizadas produce efectos positivos en las personas involucradas. Los espacios deben cumplir con la normativa educacional, deben ser cómodos, aireados, con buena iluminación natural y artificial, pintados con colores agradables a la vista, ambientados con una temperatura equilibrada, deben contener sitios de espera y descanso que permitan la tertulia y la conversación universitaria, el mobiliario debe ser ergonómico, de acuerdo a cada tarea a realizar, garantizar la comodidad del alumnado como demás personal. Es importante contar con cubículos para docentes donde los profesores atiendan a sus alumnos de una manera apropiada y cubrir así sus necesidades académicas.
6.- Servicios Adicionales. Una buena biblioteca que permita y estimule a la lectura y la investigación, servicios de internet y fotocopiadora para el público, servicios de bar donde estudiantes, personal docente y administrativo, pueda pasar sus ratos libres y de ocio, locales comerciales con precios solidarios donde se pueda surtir sin salir de la institución.

Es muy importante conocer a los estudiantes en un nivel más profundo, ya que ello es la puerta de entrada hacia el diseño de estrategias de retención efectivas y la elección de tecnologías adecuadas para gestionar la trayectoria estudiantil en la institución. Un sistema de gestión de relaciones con los estudiantes ayuda a aumentar la retención y la lealtad hacia la institución. Debemos tener presente cuál es el elemento que define la identidad institucional, tal cual menciona Lemaitre (2019, p. 25) “... que se juega en la forma en que expresa su compromiso con los estudiantes y con el conocimiento y se traduce en la definición de sus propósitos o de su entorno significativo". Un factor fundamental que apoya la retención estudiantil e impacta en la actualidad en las organizaciones, es contar como instituciones de educación superior con el dominio por parte de los docentes del uso de elementos tecnológicos. En la actualidad, la competencia digital de los alumnos y docentes conlleva a un éxito seguro en la prosecución de los objetivos educacionales. Como afirma Carbonell (2001, p. 56) "Integrar y dominar las nuevas tecnologías de la información exige una relación más interactiva entre el profesorado y el alumnado para poder intercambiar y compartir de manera más fluida $y$ permanente el acceso, la selección, la asociación y la crítica del conocimiento".

\section{CONCLUSIONES}

Las instituciones educativas se enfrentan día a día, con nuevos desafíos para cumplir con las exigencias y demandas sociales, tecnológicas, educativas, académicas y de aseguramiento estudiantil con calidad y pertinencia social. Siendo que cada institución presenta sus propias particularidades contextuales y normativas en las que se apoya para orientar el proceso formativo $y$ atender las dimensiones de gestión académica, administrativa, estudiantil e integración comunitaria y organizacional. Cada institución tiene sus propias fortalezas y debilidades para asumir el aseguramiento de la prosecución estudiantil, prevenir la deserción de los estudiantes, promover la calidad 


\section{A) Publicandous}

de la educación y aplicar estrategias dirigidas a intervenir en las siguientes dimensiones:

1. Diseño e inserción del docente en programas de formación continua.

2. Estudios sociodemográficos y socioeconómicos del estudiante y entorno familiar.

3. Estudio de factores relacionados con las instituciones educativas para mejorar la calidad educativa.

4. Integración de las tecnologías digitales y/o uso de medios tecnológicos en los currículos universitarios.

5. Digitalización de los registros de admisión y seguimiento estudiantil, tales como software de gestión y retención de estudiantes, sistemas de gestión y control al egresado.

6. Plan de marketing educativo y estrategias de aseguramiento de la calidad educativa.

7. Programas de desarrollo y atención al estudiante desde las tutorías personalizadas y el acompañamiento.

8. Plan de integración y corresponsabilidad de las autoridades educativas y miembros de la comunidad e instituciones que garanticen la inserción académica y ocupacional del estudiante desde las prácticas preprofesionales.

9. Plan de consolidación de la infraestructura, espacios y seguridad en las instalaciones de las instituciones de educación superior.

10. Actualización permanente de los currículos y diversificación de las ofertas académicas.

11. Programas de formación de investigadores e integración de la investigación al currículo.
12. Programas de orientación e inclusión a la población estudiantil que implica brindar soporte al estudiante, formulación del proyecto de vida académica y futura inserción socio profesional, asuntos académicos de enseñanza y aprendizaje.

13. Establecimiento de convenios de cooperación académica nacionales e internacionales.

14. Programas de apoyo financiero al estudiante, becas trabajo, pasantías remuneradas, estímulos por rendimiento académico, apoyo socioeconómico del estudiante en situación de vulnerabilidad.

Estas estrategias deben estar integradas en el plan de gestión educativa bajo la responsabilidad de unidades de gestión académica y administrativa en correspondencia con las políticas educativas y la misión y visión de la institución educativa. El plan estratégico de desarrollo institucional (PEDI) debe estar dirigido a retener al estudiante para garantizar su continuidad en sus estudios y posterior inserción al campo socio profesional. 


\section{REFERENCIAS BIBLIOGRÁFICAS}

Barba, T. y Heras, J. (2020) Programa de Retención Estudiantil. Documento institucional: Instituto Superior Tecnológico Particular Sudamericano: Cuenca, Ecuador.

Carbonell, J. (2001) La aventura de innovar. El cambio en la escuela. Ediciones Morata: Madrid.

\section{Google Scholar}

Gómez Figueroa en Cuevas Nava, J. L. (2000). La ANUIES en la línea del tiempo. 50 años de historia. Consultado en: https://books.google.com.ec/books/about/La_ANUI ES_en_la_1\%C3\%ADnea_del_tiempo.html?id=YdV PLtjcGPcC\&redir_esc $=y$

\section{Google Scholar}

Instituto Superior Tecnológico Particular Sudamericano (2019) Estrategias de Deserción para los Estudiantes del Instituto Superior Tecnológico Particular Sudamericano de la ciudad de Cuenca, para las Carreras de Marketing, Diseño Gráfico, Desarrollo de Software, Gastronomía y Turismo. Documento institucional. Cuenca, Ecuador.

Larrea de Granados, E. (s/f) Unidad Curricular de Titulación. Documento de apoyo no vinculante. Consejo de Educación Superior (CES) Recuperado en https://www.ces.gob.ec/doc/2-seminario/unidadde-titulacion.pdf

\section{Google Scholar}

Lemaitre, M. (2019) Aseguramiento de la calidad en América Latina. En: Aseguramiento de la calidad de la educación superior. Debates y experiencias, 2019. Consejo de Aseguramiento de la Calidad de la Educación Superior: Quito, Ecuador.
Pineda-Báez, C., Pedraza-Ortiz, A., \& Moreno, I. (2011). Efectividad de las estrategias de retención universitaria: la función del docente.Educación y Educadores, 14(1). Recuperado de https://educacionyeducadores.unisabana.edu.co/index. php/eye/article/view/1833/2411

\section{Google Scholar}

Segunda Cumbre de Las Américas (1998). Plan de Acción Suscripto por los Jefes de Estado y de Gobierno Asistentes a la Segunda Cumbre de las Américas. México: Secretaría de Educación Pública.

Swail, W. S. (2003). Retaining Minority Students in Higher Education: A Framework for Success. ASHE-ERIC Higher Education Report. Jossey-Bass Higher and Adult Education Series. Jossey-Bass, 989 Market Street, San Francisco, CA 94103-1741.

\section{Google Scholar}

U-Planner, (2019) ¿Cómo Mejorar la Retención Estudiantil en la Educación Superior en EEUU? Nuevas Estrategias, Enfoques y Tecnologías para atraer y retener a los Estudiantes. Recuperado de https://www.u-planner.com/como-mejorar-lastasas-de-retencion-de-estudiantes-en-laeducacion-superior-de-estados-unidos

Viera Orozco, J. J. (2016). Estrategias de comunicación interna del GADM-C guano y su incidencia en el desarrollo institucional, período julio a diciembre de 2013 (Bachelor's thesis, Riobamba: Universidad Nacional de Chimborazo, 2016.).

\section{Google Scholar}

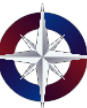

